\title{
Uninstructed Participants Use Pain Scales To Indicate Social Distress
}

\author{
Norma Rosenek ${ }^{1}$ | Stella Vagena ${ }^{1}$ | India Winterbourne ${ }^{1}$ | Carien van Reekum ${ }^{1}$ | Amanda C. Williams ${ }^{2}$ | Tim V. Salomons ${ }^{1,3}$ \\ ${ }^{1}$ Department for Psychology and Clinical Language Sciences, University of Reading, UK; ${ }^{2}$ Research Department of Clinical, Educational \& Health Psychology, UCL, UK; ${ }^{3}$ \\ Department of Psychology, Queen's University, Kingston (ON), Canada
}

\section{Background}

The International Association for the Study of Pain (IASP) defines pain as "an unpleasant sensory and emotional experience associated with actual or potential tissue damage, or described in terms of such damage" ${ }^{\prime 2}$.

In common usage, the term "pain" is often used to denote emotional distress. In recent years, this broader use of the term has also permeated the scientific literature, notably to describe the distress of social exclusion ("social pain") ${ }^{2}$. This raises the possibility that individuals might use pain scales to reflect their current emotional state, creating ambiguity around self-reported pain in clinical and experimental settings.

To examine this possibility, we elicited social distress and asked participants to rate pain both with and without explicit instructions on what should be considered pain.

\section{Method}

\section{Participants}

Forty healthy subjects ( 23 females / 17 males; age: $24 \pm 8$ ) participated and were split into instructed $(n=20)$ vs. uninstructed $(n=20)$ groups.

\section{Procedure}

Instructed group

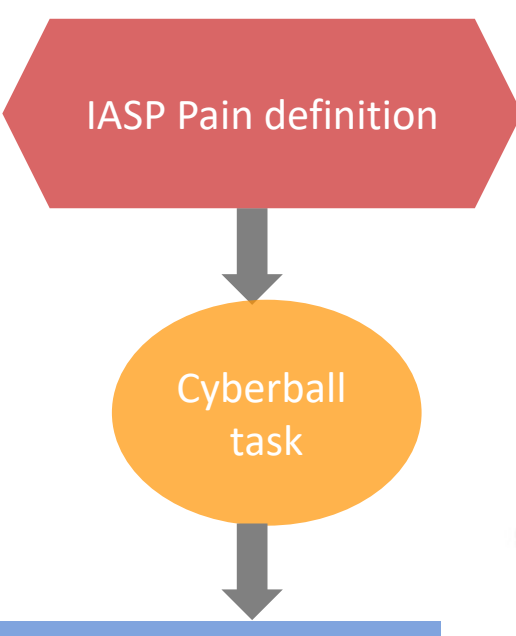

Ratings: Ratings:
Pain \& Unpleasantness

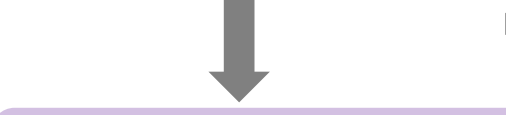

Uninstructed group

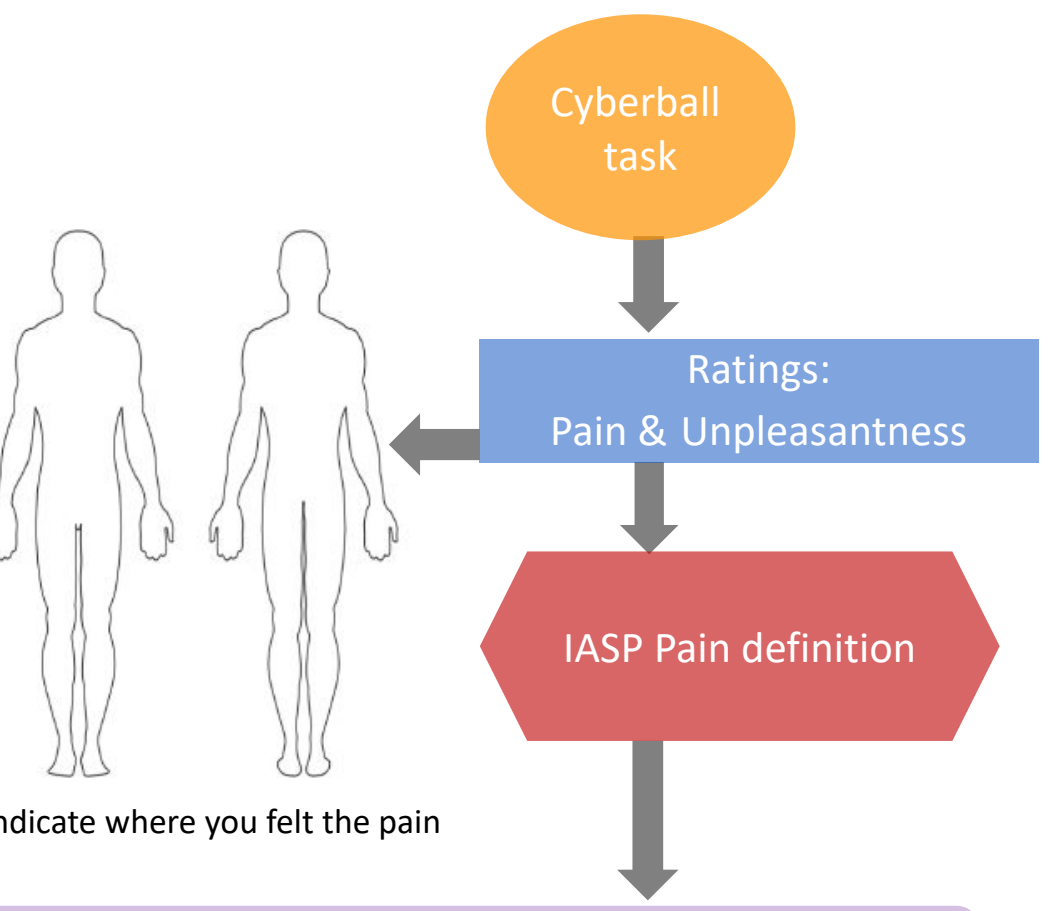

Follow up questions

\section{Cyberball task}

Participants played a computerised ball-tossing game (Cyberball 5.0) in a threeplayer social exclusion paradigm. Initially, they received the ball twice, but were excluded on all subsequent 40 throws.
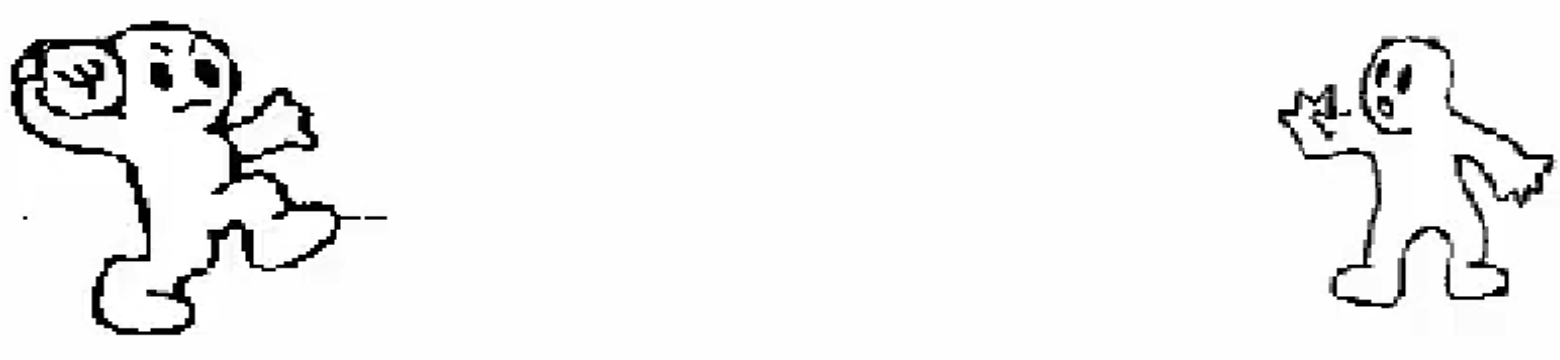

\section{Results}

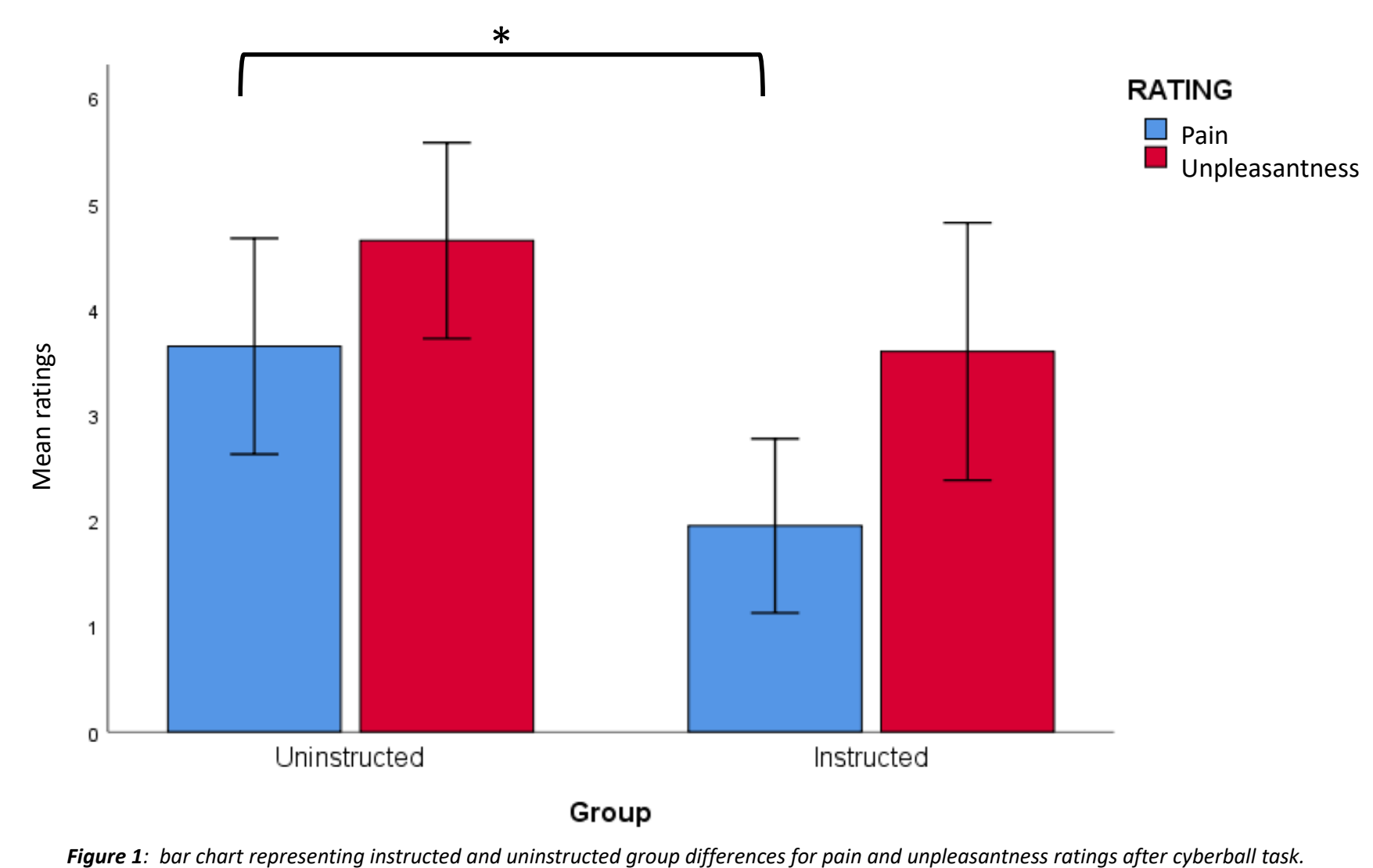

Despite receiving no nociceptive stimulation, participants rated the cyberball experience as painful, implying that "pain" was being used to indicate a negative emotional state. This tendency was significantly reduced for individuals given the IASP definition prior to the cyberball task ( $M=3.65$ for uninstructed individuals, $M=1.95$ for instructed; $t=2.18, p<0.01$ ).

In contrast, regardless of prior instructions of the pain definition, participants rated the experience similarly unpleasant $(M=4.65$ for uninstructed individuals, $M=3.60$ for instructed; $t=1.44, p=0.16)$.

\section{Follow up questions}

Participants were given the following multiple choice questions to describe the pain experience further.

$$
\begin{array}{ll}
\text { The pain } & \text { The pain I indicated on the body map felt like } \\
\text { - Felt like an injury to my body } & \text { - Physical pain that feels like an injury } \\
\text { - Made me feel rejected and/or sad } & \text { - Emotional pain }
\end{array}
$$

Out of 40 participants, none reported that they perceived the pain as "injury to their body" or "physical pain that feels like an injury".

The experience made $82.5 \%$ "feeling sad or rejected" and was described as "emotional pain" by $75 \%$.

\section{Conclusion}

> These results indicate that participants will use pain scales to rate their emotional state, even in the absence of any nociceptive stimulation.

$>$ This behaviour was significantly reduced by providing explicit instructions about what should or shouldn't be called pain. 